\title{
On Drawing Angle Graphs ${ }^{\star}$
}

\author{
(Extended Abstract)
}

\author{
Ashim Garg \\ Department of Computer Science \\ Brown University \\ Providence, RI 02912-1910, USA
}

\begin{abstract}
An Angle graph is a graph with a fixed cyclic order of edges around each vertex and an angle specified for every pair of consecutive edges incident on each vertex. We study the problem of constructing a drawing of an angle graph that preserves its angles, and present several new results.
\end{abstract}

\section{Introduction}

An angle graph is a graph with a fixed cyclic order of edges around each vertex and an angle (between $0^{\circ}$ and $360^{\circ}$ ) specified for every pair of consecutive edges incident on each vertex such that the sum of angles around every vertex is $360^{\circ}$. A Rectilinear angle graph is an angle graph in which each angle is a multiple of $90^{\circ}$.

A graph drawing algorithm takes a graph as its input and constructs a drawing of the graph (see [3] for an extensive survey). Some well known algorithms such as Tamassia's bend-minimization algorithm [16] and the visibility representation algorithm by Tamassia and Tollis [17] use rectilinear angle graphs as intermediate stage products. Infact the bend-minimization algorithm has been found to give aesthetically pleasing drawings in practice [11]. Our hope is that a good characterization of planar angle graphs may allow us to use the bend minimization algorithm to construct aesthetically pleasing drawings of any planar graph, not just orthogonal planar graphs.

A recent trend in the area of graph drawing is towards developing systems which allow user to specify arbitrary constraints on the positioning of vertices and edges in the drawings. See for example, $[2,6,12]$. Since a user may specify angle constraints, a study of angle graphs is important for such systems.

A very important aesthetic criteria for a straight line drawing (edges drawn as straight lines) is that the angles between the consecutive edges incident on each vertex be large. This is formalized by defining angular resolution of a drawing [7, 15]. Angular resolution of a straight line drawing of a graph is the minimum angle in the drawing between any two consecutive edges incident on the same vertex.

Studying angle graphs can provide important clues in constructing drawings with large angular resolution. For example we use our result, that testing an angle graph for planarity is $N P$-hard, to show that, given a triconnected planar graph $G$ and an angle $\alpha$, determining whether $G$ admits a planar straight line drawing with angular resolution at least $\alpha$ is $N P$-hard.

Angles have been found to be useful in characterizing planar graphs (see [5], for example). The study of angle graphs is also of theoretical interest in itself

\footnotetext{
* Research supported in part by the National Science Foundation, by the U.S. Army Research Office, by the Office of Naval Research and the Defense Advanced Research Projects Agency.
} 
because they require an extensive use of plane geometry in their analysis. Most of the known graph drawing algorithms use graph theoretic concepts such as orientation, coloring, flow etc. Recent results [9] however shows that plane geometry is a useful tool by successfully applying geometric techniques in deriving many results on planar drawings with large angular resolution. A study of angle graphs can provide an invaluable insight into the use of plane geometry for graph drawing algorithms and stimulate research in this relatively unexplored area.

We now give some definitions. Let $A$ be an angle graph. A drawing of $A$ is a mapping of its vertices to points in the plane and its edges to straight lines joining their endpoints so that the angles of $A$ are preserved in the drawing. A planar drawing of $A$ has no edge crossings. Unlike general graphs, an angle graph may not have a drawing at all. Fig $1(a)$ shows such an angle graph (the angles between edges are as given in the figure). Fig $1(b)$ shows an angle graph that has a drawing. $A$ is called consistent if it has a drawing. $A$ is called planar if it has a planar drawing. The graph isomorphic to $A$ if we drop the angle constraints is called the underlying graph of $A$. An angle-cycle (outerplanar angle graph, series-parallel angle graph, resp.) is an angle graph whose underlying graph is a cycle (outerplanar graph, series-parallel graph, resp.).

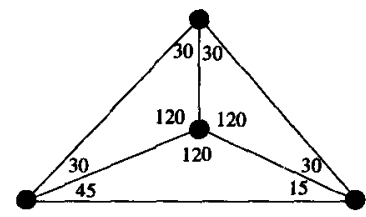

(a)

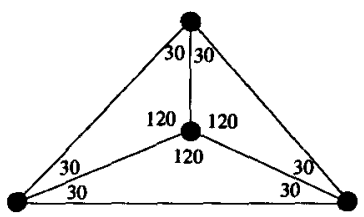

(b)

Figure 1: An angle graph (a) that is not consistent; (b) that is consistent.

The pioneering work in the area of angle graphs was done by Vijayan in [19]. Vijayan gave a linear program for testing an angle graph for consistency. Some necessary conditions for an angle graph to be planar, as well as necessary and sufficient conditions for some special angle graphs such as angle cycles, angle graphs with convex faces, outer planar angle graphs and rectilinear angle graphs have also been described in [19]. Vijayan also posed some conjectures about the planarity of the angle graphs. A characterization of planar angle graphs with triangular faces by a set of non-linear equalities is given in [5]. [20] gives a linear time algorithm for testing a rectilinear angle graph for planarity and quadratic time algorithm for constructing a planar drawing if it is planar.

\subsection{Our Results}

We give several new results concerning drawing of angle graphs. We first study the planarity of angle graphs. We disprove the conjectures of [19] by providing counter examples to them. We then show that testing a consistent angle graph for planarity is $N P$-hard. We also show that given a triconnected planar graph and an angle $\alpha$, determining whether it admits a planar straight line drawing with angular resolution at least $\alpha$ is $N P$-hard. Our result therefore strengthens a similar result given by Kant [13] that holds for biconnected planar graphs. 
We study the problem of testing a series-parallel angle graph for consistency and provide a linear time algorithm for solving the problem.

The study of area requirements of drawings of graphs has received a lot of attention (see, e.g., [1, 4, 8, 9]) and is motivated by the finite resolution of the graph drawing technologies and circuit-area optimization criteria of VLSI layouts $[14,18]$. We study the area requirement of angle graphs and show that there exists a family of angle graphs requiring exponential area in drawing.

Finally we consider the multiplanarity problem of angle graphs. A multilayered angle graph is an angle graph in which each edge is assigned to one of several layers. A multiplanar angle graph is a multilayered angle graph if it has a drawing in which edges assigned to the same layer do not cross. We show that very surprisingly testing even a bilayered rectilinear angle graph (edges assigned to only two layers) for biplanarity is $N P$-hard. This is in sharp contrast to the linear time complexity of testing a monolayer rectilinear graph for planarity [20].

\section{Planarity Testing of Angle Graphs}

Vijayan [19] made following conjectures (see [19] for definitions of the terms used in the conjecture. We do not describe them here for the lack of space):

Conjecture 1 (Vijayan [19]) A consistent angle graph $A$ that does not contain any angle-cycle which is a subdivision of a triangle is planar if and only if (a) the faces of each biconnected component are consistent angle-cycles, $(b)$ no two biconnected components interlace at an articulation vertex of $A$, and (c) the force inside relation among the biconnected components is a partial order.

Conjecture 2 (Vijayan [19]) The conditions stated in the Conjecture 1 are necessary and sufficient for consistent angle graphs that do not contain subdivisions of triangles and whose biconnected components have convex interior faces.

We give a counter example to Conjecture 2, which in turns disproves Conjecture 1. Consider the angle graph $A$ shown in Fig. 2(a). $u$ is an articulation vertex of $A$. $A$ has two biconnected components $B_{1}$ and $B_{2}$. Both $B_{1}$ and $B_{2}$ have convex interior faces and angle graph $A$ does not contain subdivisions of triangles. By simple geometric considerations we can show that $A$ is not planar.

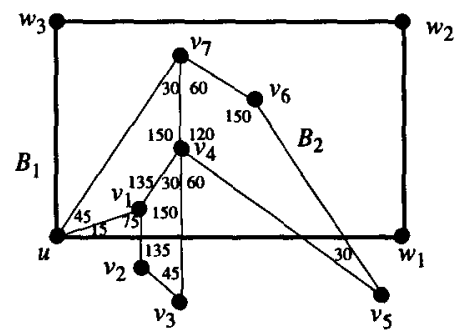

Figure 2: Counter example to Conjecture 2: Angle graph $A$.

\subsection{NP-Hardness of Planarity Testing}

We now show that testing a consistent angle graph for planarity is $N P$-hard by reducing the following version of the $3 S A T$ problem to it: 
given a set $X=\left\{x_{1}, x_{2}, \ldots, x_{n}\right\}$ of variables and a set $C=$ $\left\{c_{1}, c_{2}, \ldots, c_{m}\right\}$ of clauses over $X$ such that every clause has three literals, each variable occurs in at most five clauses either negated or unnegated [10] and in none of them both as negated and un-negated, is there a satisfying truth assignment for $C$ ?

We construct an angle graph $A$ such that there is a satisfying truth assignment of $C$ if and only if $A$ is planar. We need the following gadgets (which are angle graphs) for our construction. We denote the distance between two vertices $u$ and $v$ in a drawing of $A$ by $d(u, v)$.

- Horse shoe: We use the horse shoe to represent the variables. A horse shoe $H$ is shown in Fig. $3(a)$. The vertices $\left\{x_{0}, x_{1}, \ldots, x_{9}\right\}$ and $\left\{y_{0}, y_{1}, \ldots, y_{9}\right\}$ are called the output vertices of the faces $F_{x}$ and $F_{y}$ respectively. Vertices $x_{i}$ and $x_{i+1}\left(y_{i}\right.$ and $\left.y_{i+1}\right)$ for even values of $i$ are siblings of each other. Edges $e_{x}, e_{y}$ and $e_{t}$ are called the left attachment, right attachment and top edge respectively of the horse shoe. In any planar drawing $\Delta$ of $H$.

Observation 1: the attachments of $H$ have same length as its top edge.

Observation 2: if the top edge of $H$ has unit length then at most one of $d\left(x_{0}, x_{9}\right)$ and $d\left(y_{0}, y_{9}\right)$ is at least one unit. $H$ is left heavy in $\Delta$ if $d\left(x_{0}, x_{9}\right) \geq 1$ (Fig. 3(b)) and is right heavy in $\Delta$ if $d\left(y_{0}, y_{9}\right) \geq 1$ (Fig. 3(c)).

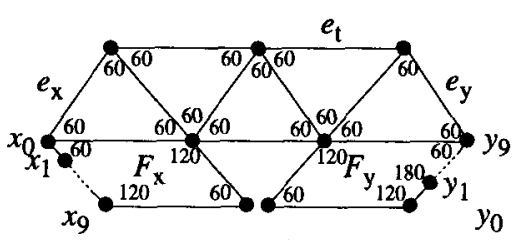

(a)

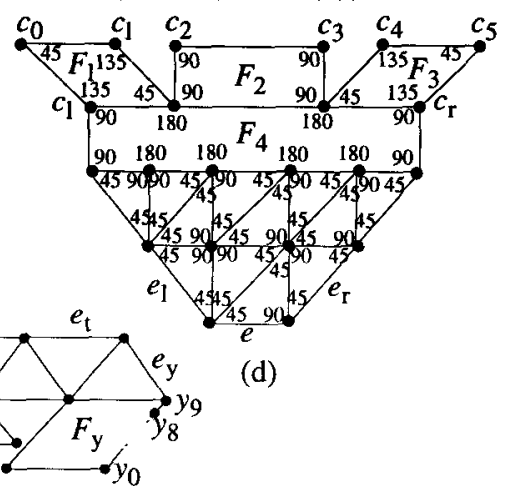

(c)

Figure 3: (a) A horse shoe $H$; (b) Left heavy horse shoe; (c) Right heavy horse shoe; (d) A Crown.

- Crown: We use the crown to represent the clauses. Fig. $3(d)$ shows a crown. Edges $e_{l}$ and $e_{r}$ are called the left attachment and right attachment respectively of the crown. Edge $e$ is the base of the crown.

Observation 3: If the base has unit length then $\sum_{0 \leq i \leq 2} d\left(c_{2 i}, c_{2 i+1}\right)=5$.

- Beam: A Beam $B$ is shown in Fig. 4(a). A beam consists of 123 equilateral triangles. Edges $e_{l}$ and $e_{r}$ are called the left attachment and right attachment respectively of the beam. In any planar drawing of $B$,

Observation 4: All its edges are of equal length.

Observation 5: $d(u, v)=61$ if the length of an attachment is one unit.

- Wiggle: A wiggle $W$ is shown in Fig. $4(b)$. Vertices $u_{1}$ and $u_{2}\left(v_{1}\right.$ and $\left.v_{2}\right)$ are called the input (output) vertices of the wiggle. In any planar drawing of $W$, 
Observation 6: The horizontal distance between its output vertices equals the vertical distance between its input vertices.

Observation 7: The structure of this gadget does not place any restrictions on the horizontal distance between its input and output vertices.

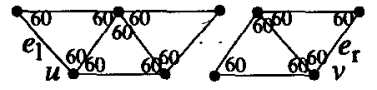

(a)

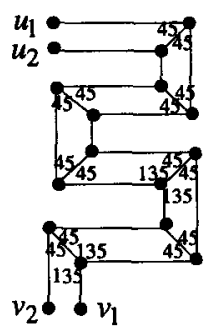

(b)

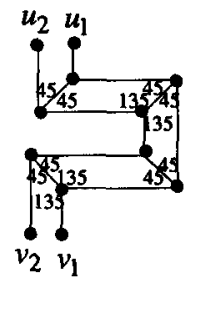

(c)

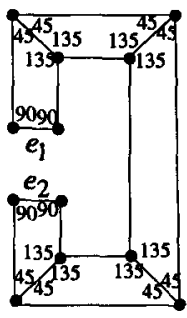

(d)

Figure 4: (a) A Beam; (b) A Wiggle; (c) A Connector; (d) A Holder.

- Connector: A connector $C$ is a subdivision of the angle graph shown in Fig. $4(c)$. Vertices $u_{1}$ and $u_{2}\left(v_{1}\right.$ and $\left.v_{2}\right)$ are called the input (output) vertices of $C$. In any planar drawing of $C$,

Observation 8: The horizontal distance between its output vertices equals the horizontal distance between its input vertices.

Observation 9: Same as Observation 7.

- Holder: A holder $H$ is shown in Fig. 4(d). Edges $e_{1}$ and $e_{2}$ are called its upper clamp and lower clamp respectively. In any planar drawing of $H$, Observation 10: The upper and lower clamps of $H$ are of same length.

Fig 5(a) gives a high level view of $A$. We can identify three important subgraphs of $A$, namely variable subgraph, connection subgraph and clause subgraph. The variable and connection subgraphs are "connected" through some wiggles, the connection and clause subgraphs are "connected" through some wiggles. The variable and clause subgraphs are "connected" through a single holder $R$.

The variable subgraph $V_{n}$ of $A$ can be described recursively: $V_{1}$ is a horse shoe $X_{1} ; V_{n}$ is constructed from $V_{n-1}$ by "connecting" the horse shoe $X_{n-1}$ (present in $V_{n-1}$ ) with a horse shoe $X_{n}$ using a beam $B$ as shown in Fig 6(a). We say that horse shoe $X_{i}$ represents variable $x_{i}$.

The clause subgraph $S_{m}$ of $A$ can be described recursively: $S_{1}$ is a crown $C_{1} ; S_{m}$ is constructed from $S_{m-1}$ by "connecting" the crown $C_{m-1}$ (present in $S_{m-1}$ ) with a crown $C_{m}$ using a beam $B$ and two edges $e^{\prime}$ and $e^{\prime \prime}$ as shown in Fig 6(b). We say that crown $C_{j}$ represents clause $c_{j}$.

The connection subgraph $D$ is constructed in following steps:

1. Let $G$ be a bipartite graph whose vertices can be partitioned into two sets $P$ and $Q$. There is a vertex $x_{i, j}$ in $P$ if literal $x_{i}$ occurs in clause $c_{j}$. There is a vertex $y_{i, j}$ in $P$ if literal $\bar{x}_{i}$ occurs in clause $c_{j} . x_{i, j}\left(y_{i, j}\right)$ is called an image of the literal $x_{i}\left(\overline{x_{i}}\right) . Q$ has a vertex $c_{j, i}$ if either literal $x_{i}$ or $\overline{x_{i}}$ occurs in clause $c_{j}$ (notice that both of them can not occur simultaneously in $c_{j}$ ). $c_{j, i}$ is called an image of clause $c_{j}$. There is an edge in $G$ between $x_{i, j}$ (or $y_{i, j}$ ) and $c_{j, i}$. Construct a drawing $\Delta$ as shown in Fig. $7(a)$ in which the vertices 


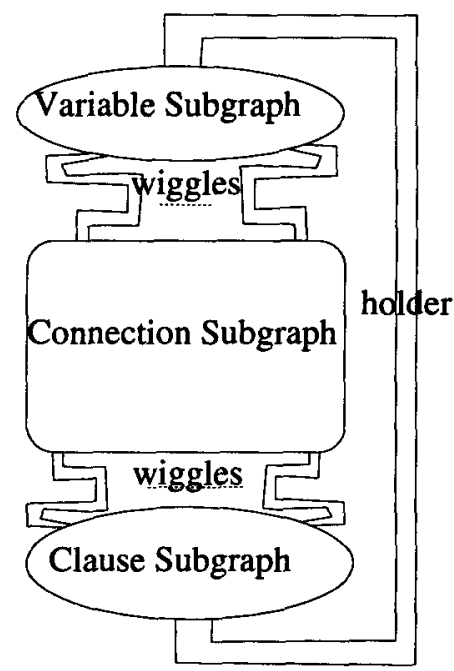

(a)

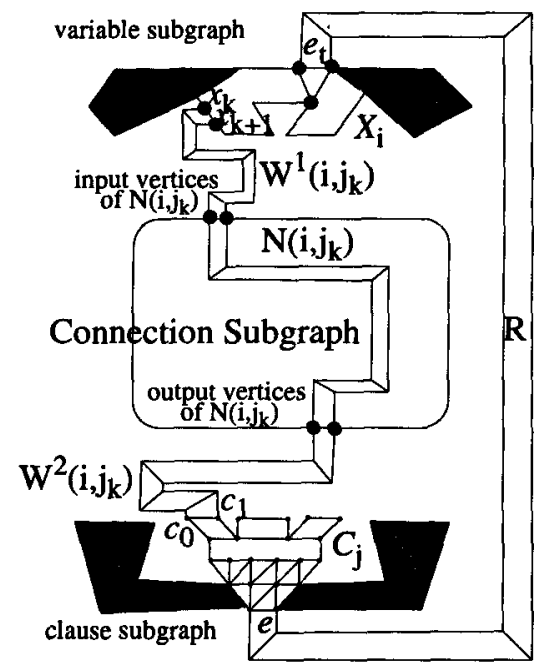

(b)

Figure 5: (a) A High Level View of the Angle Graph $A ;(b)$ Constructing angle graph $A$ : "connecting" siblings $x_{k}$ and $x_{k+1}$ in horse shoe $X_{z}$ with $N\left(i, j_{k}\right)$ by wiggle $W^{1}\left(i, j_{k}\right)$, "connecting" $c_{0}$ and $c_{1}$ in crown $C_{j}$ with $N\left(i, j_{k}\right)$ by wiggle $W^{2}\left(i, j_{k}\right)$ and "connecting" Horse shoe $X_{\imath}$ with Crown $C_{\jmath}$ with a Holder $R$.

of $P$ are placed on the same horizontal level and so are the vertices of $Q$.

2. Replace each line-segment $\left(x_{i, j}, c_{j, i}\right)$ or $\left(y_{i, j}, c_{j, i}\right)$ in $\Delta$ by the drawing of a connector $N(i, j)$ (this also means replacing $x_{i, j}$ and $c_{j, i}$ by the input and output vertices resp. of $N(i, j))$. Fig. $7(b)$ shows the resultant drawing $\Delta^{\prime}$.

3. Construct a planar angle graph $D$ by replacing the crossings in $\Delta^{\prime}$ by vertices.

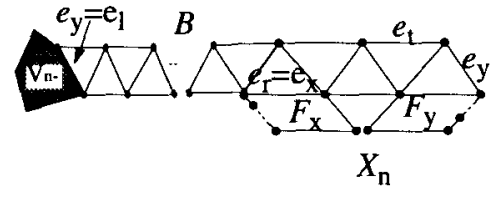

(a)

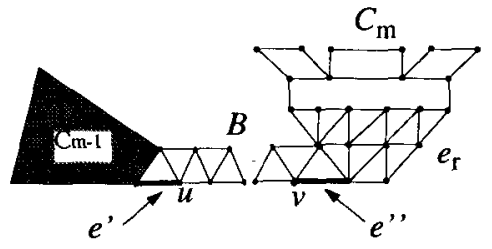

(b)

Figure 6: (a) Attaching $V_{n-1}$ and a Horse shoe $X_{n}$ to Construct Variable Subgraph $V_{n}$; (b) Attaching $S_{m-1}$ and a Crown $C_{m}$ to Construct Clause Subgraph $S_{m}$.

Angle Graph $\mathbf{A}$ We now complete the description of angle graph $A$.

- Suppose literal $l_{i}$ where $l=x$ or $l=y=\bar{x}$ occurs in clauses $c_{j_{0}}, c_{j_{1}}, \ldots, c_{j_{r}}$, where $j_{k}<j_{k+1}$ and $r<5$. "Connect" siblings $l_{k}$ and $l_{k+1}$ in horse shoe $X_{i}$ (recall that $X_{i}$ represents variable $x_{i}$ ) with connector $N\left(i, j_{k}\right)$ using a wiggle $W^{1}\left(i, j_{k}\right)$ (see Fig. $5(b)$ which shows connection of $x_{k}$ and $x_{k+1}$ ). Edge $\left(l_{k}, l_{k+1}\right)$ is called the image of clause $c_{j_{k}}$ in $X_{i}$.

- Suppose variables $x_{i_{0}}, x_{i_{1}}$, and $x_{i_{2}}$ where $i_{0}<i_{1}<i_{2}$ occur in a clause $c_{j}$ either negated or un-negated. "Connect" $c_{2 k}$ and $c_{2 k+1}$ in crown $C_{j}$ with 


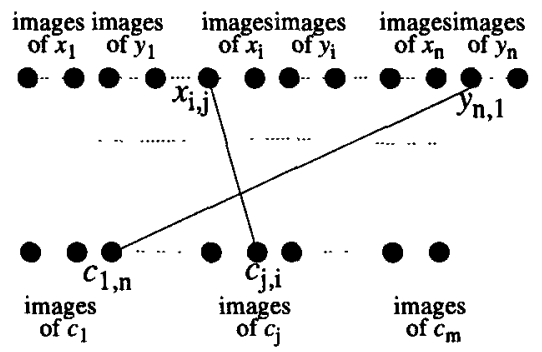

(a)

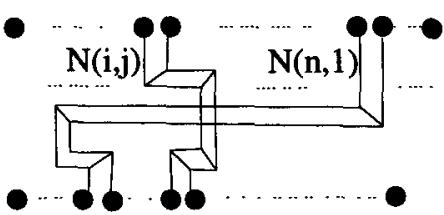

(b)

Figure 7: Bipartite graph $G$ : (a) A drawing $\Delta$ of $G$; (b) Drawing $\Delta^{\prime}$. connector $N\left(i, j_{k}\right)$ using a wiggle $W^{2}\left(i, j_{k}\right)$ (Fig. $5(b)$ shows connection with $c_{0}$ and $\left.c_{1}\right)$. Edge $\left(c_{k}, c_{k+1}\right)$ is called the image of variable $x_{i_{k}}$ in $C_{j}$.

- Now choose any horse shoe $X_{i}$ and crown $C_{j}$, and "connect" $X_{i}$ with $C_{j}$ by identifying the upper clamp of a holder $R$ with the top edge of $X_{i}$ and the lower clamp of $R$ with the base of $C_{j}$ (see Fig. $5(b)$ ).

Theorem 1. There is a satisfying truth assignment of $C$ if and only if $A$ is planar.

\section{Sketch of Proof:}

If: Suppose $A$ is planar. Let $\Delta$ be a planar drawing of $A$. Let the clamps of the holder have unit length in $\Delta$. Every $X_{i}$ is either left heavy or right heavy in $\Delta$ (Observation 2). Set variable $x_{i}$ to true if $X_{i}$ is left heavy and to false otherwise. Suppose variable $x_{i}$ occurs in clause $c_{j}$. From Observations 6 and 8 , the length of the image of $x_{i}$ in $C_{j}$ is equal to $\sqrt{3} / 2$ times the length of the image of $c_{j}$ in $X_{i}$. Therefore the image of $x_{i}$ in $C_{j}$ has at least $\sqrt{3} / 2$ units length only if literal $x_{i}$ or $\bar{x}_{i}$ that occurs in $c_{j}$ is true. From Observation 3 at least one of the images of the variables occurring in $C_{j}$ has length at least $\sqrt{3} / 2$ units. Consequently $C_{j}$ is satisfiable.

Only If: It is easy to see that because the length of a beam is at least 61 units in any drawing of $A$, (the length of the clamps of the holder is taken to be one unit), the greater of $d\left(x_{0}, x_{9}\right)$ and $d\left(y_{0}, y_{9}\right)$ in any $X_{i}$ can be $5(2 / \sqrt{3})$ units without creating any crossings. Wiggles (Observation 7) provide enough flexibility to construct a planar drawing for $A$.

Theorem 2. The problem of testing whether a consistent angle graph is planar is NP-hard.

All the angles used in our reduction were multiples of $15^{\circ}$. Therefore,

Corollary 3. The problem of testing whether a consistent angle graph is planar is NP-hard even if the angles specified for every pair of consecutive edges incident on a vertex in the angle graph is a multiple of some integer $\alpha \neq 0$.

\section{Testing Triconnected Planar Graphs for High Angular Resolution}

We now consider the problem of constructing planar straight line drawings of planar graphs with high angular resolution and show that the following problem is NP-hard: 
Given a triconnected planar graph $G$ and an angle $\alpha$, determine whether $G$ has a planar straight line drawing with angular resolution at least $\alpha$.

We use the consistent angle graph $A$ described in Section 2.1 to show that this problem is $N P$-hard. Let $H$ be the underlying graph of $A$. Our approach is to convert $H$ into a triconnected planar graph $G$ in polynomial time by adding some special gadgets called fans that are described by Kant in [13], such that $G$ has a planar straight line drawing with angular resolution at least $5^{\circ}$ if and only if $A$ is planar. Details are provided in the full paper.

Theorem 4. Given a triconnected planar graph and an angle $\alpha$, determining whether it admits a planar straight line drawing with angular resolution at least $\alpha$ is NP-hard.

\section{Drawing a Series-Parallel Angle Graph}

A series-parallel directed graph $G$ with a source and a sink [1] is defined recursively as follows: A series-parallel directed graph is either a single directed edge or a series composition or a parallel composition of two series-parallel directed graphs $G_{1}$ and $G_{2} . G_{1}$ and $G_{2}$ are called the series-parallel components of $G$.

We show that a series-parallel can be tested for consistency in linear time. Our approach is bottom up: We assume a cartesian coordinate system in which left, right, above and below have their usual meanings. Hence assuming that the source of the series-parallel graph is placed at the origin, we compute the range over all the possible drawings of the graph, of the angles made with the $x$-axis by an imaginary line-segment joining its source and sink. This information is encoded in form of two tuples $(\alpha, \beta)$ and $(\gamma, \delta) . \alpha$ and $\beta(\gamma$ and $\delta)$ are the lower and upper bounds on the angles made by this imaginary line-segment in all the drawings in which the sink is placed to the left (right) of the source. These tuples are computed for a series-parallel graph $G$ in constant time from the tuples of its series-parallel components, giving a linear time testing algorithm. This approach can be easily modified to give a linear time algorithm for drawing a series-parallel angle graph. The details of this approach are provided in the full paper.

Theorem 5. Given a series-parallel angle graph wath $n$ vertices, we can test if it admits a drawing or not and construct a drawing if it does in $O(n)$ time.

\section{Area Requirement of Angle Graphs}

We now investigate the area requirement of angle graphs. Our main theorem is:

Theorem 6. For every $m \geq 1$, there exısts an angle graph $A_{m}$ with $3 m$ vertices that requires area $\Omega\left(4^{m}\right)$ for any drawing.

Sketch of Proof: Angle graph $A_{m}$ can be described recursively: Angle graph $A_{1}$ is shown in Fig. 8(a). Angle graph $A_{m}$ is constructed from $A_{m-1}$ (see Fig $8(b)$ ) by introducing vertices $u_{m}, v_{m}$ and $w_{m}$ and edges $\left(u_{m-1}, u_{m}\right),\left(v_{m-1}, u_{m}\right)$, $\left(v_{m-1}, v_{m}\right),\left(w_{m-1}, v_{m}\right),\left(w_{m-1}, w_{m}\right)$ and $\left(u_{m-1}, w_{m}\right)$ in the exterior of the angle graph $A_{m-1}$.

Using plane geometry we show that Area $\left(A_{m}\right)=4$ Area $\left(A_{m-1}\right)$ and consequently Area $\left(A_{m}\right)=\Omega\left(4^{m}\right)$. 


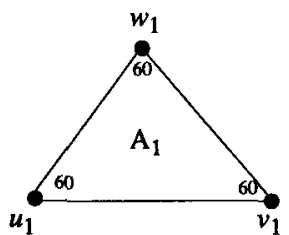

(a)

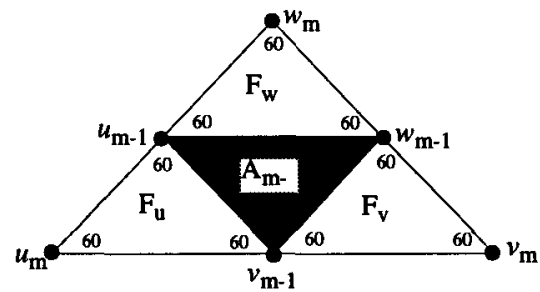

(b)

Figure 8: Angle graph $A_{m}:(a) A_{1} ;(b)$ Constructing angle graph $A_{m}$ from $A_{m-1}$.

\section{Multilayered Angle Graphs}

Recall the definition of multilayered angle graphs and multiplanar angle graphs from Sec. 1.1. We now show that even if we consider only rectilinear graphs and restrict the number of layers to two, the problem of testing them for biplanarity is NP-hard.

We reduce the 3 SAT problem with restriction that a variable can not occur both negated and un-negated in the same clause, to an instance of this problem. Suppose we are given a set $X=\left\{x_{1}, x_{2}, \ldots, x_{n}\right\}$ of variables and a set $C=$ $\left\{c_{1}, c_{2}, \ldots, c_{m}\right\}$ of clauses. We construct a bilayered rectilinear angle graph $A$ with two layers called the red and blue layers such that there is a satisfiability assignment of $C$ if and only if $A$ is biplanar. We call the edges of $A$ belonging to the red (blue) layer as red (blue) edges. We use the following gadgets (which are bilayered angle graphs) for constructing $A$ :

- Subcourse: A subcourse $S$ is shown in Fig $9(a)$. Vertex $u_{j}$, where $1 \leq j \leq$ $2 m$ is called the $j^{t h}$ input vertex of $S$. Vertex $v_{j}$, where $1 \leq j \leq 2 m$ is called the $j^{\text {th }}$ output vertex of $S$. Edge $\left(t_{j}, t_{j+1}\right)$, where $1 \leq j \leq m$ is called the $j^{\text {th }}$ reference edge of $S$. Edge $\left(s_{j}, s_{j+1}\right)$, where $1 \leq j \leq m$ is called the $j^{\text {th }}$ value edge of $S$. Each reference as well as value edge of $S$ can either be blue or red (as will be described later). Similarly edges $\left(d_{1}, d_{2}\right)$ and $\left(d_{3}, d_{4}\right)$, called the top clamping and bottom clamping edges of $S$ respectively, can be either blue or red. Edge $\left(a_{2 j-1}, a_{2 j}\right)$ for $1 \leq j \leq m$ is called the $j^{\text {th }}$ inner edge of $S$ and is blue. Edge $\left(v_{2 j-1}, v_{2 j}\right)$ for $1 \leq j \leq m$ is called the $j^{\text {th }}$ outer edge of $S$ and is red. Each edge $\left(u_{2 j}, a_{2 j}\right)$ as well as each edge $\left(a_{2 j}, v_{2 j}\right)$, where $1 \leq j \leq m$ is red. Other edges of the subcourse are blue.

Let $\Delta$ be a drawing of $S$. The vertical line segment $\left(s_{1}, s_{m+1}\right)$ is called the value line segment of $S$ in $\Delta$. The vertical line segment $\left(t_{1}, t_{m+1}\right)$ in $\Delta$ is called the reference line segment of $S$ in $\Delta$. The value line segment is either to the right of the reference line segment (Fig. $9(b)$ ) or it is to the left of the reference line segment(see Fig. $9(c)$ ) or both overlap. If the value line segment is to the left (right) then $S$ is said to be left heavy (right heavy) in $\Delta$. If value and reference line segments overlap then $S$ is balanced in $\Delta$. As we will see later, each subcourse corresponds to a variable and the left heavy (right heavy) "state" corresponds to the variable being true (false).

- Starting and Ending Location: Fig. 10(a) shows a starting location $L$ and Fig. 10(b) shows an ending location $L^{\prime}$. Vertex $c_{j}\left(c_{j}^{\prime}\right)$, where $1 \leq j \leq m$ is called the $j^{t h}$ starting (ending) vertex of $L\left(L^{\prime}\right)$. Vertex $w_{j}$, where $1 \leq$ 

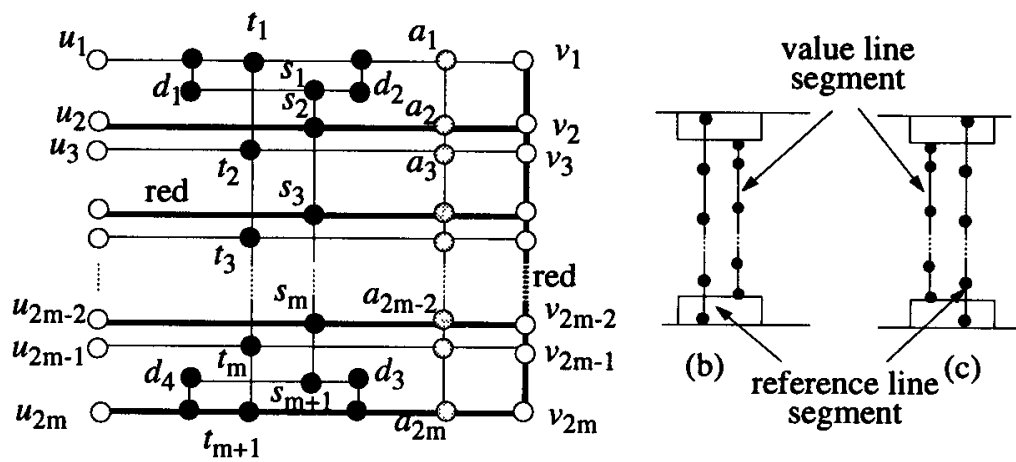

(a)

Figure 9: (a) A subcourse $S$; (b) A left heavy subcourse; (c) A right heavy subcourse.

$j \leq 2 m$ is called the $j^{t h}$ separation vertex of $L\left(L^{\prime}\right)$. Vertices $c_{1}, c_{2}, \ldots, c_{m}$ $\left(c_{1}^{\prime}, c_{2}^{\prime}, \ldots, c_{m}^{\prime}\right)$ are called the starting (ending) vertices of the starting (ending) location. All the edges of both starting and ending locations are blue.

- Hurdle: A hurdle $H$ is shown in Fig. 10(c). Vertex $g_{j}$ where $1 \leq j \leq 2 m$, is the $j^{\text {th }}$ input vertex of $H$. Vertex $h_{j}$, where $1 \leq j \leq 2 m$ is the $j^{\text {th }}$ output vertex of $H$. Each edge $\left(h_{j}, h_{j+1}\right)$ is red. Other edges of the hurdle are blue.

- Runner: A runner $R($ Fig. $10(d))$ is a connected sequence of 14 alternating red and blue edges. The angle between consecutive edges is $180^{\circ}$. Vertex $a$ is the first end point of $R$ and vertex $a^{\prime}$ is its last end point. The edge incident on $a$ and $a^{\prime}$ are called the first and last edges of $R$ respectively. The first edge of $R$ is blue. As we will see later each runner corresponds to a clause.

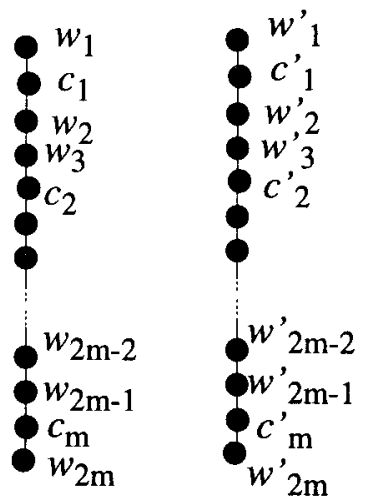

(a) (b)

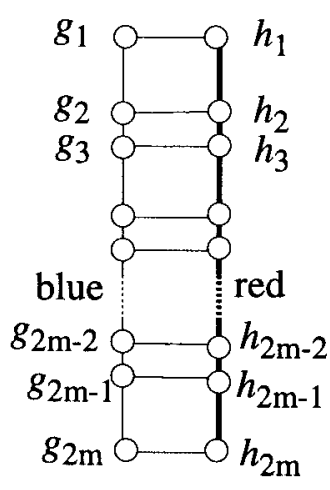

(c)

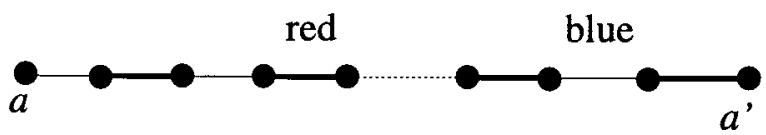

(d)

Figure 10: (a) A Starting Location; (b) An Ending Location; (c) A Hurdle; (d) A Runner. 


\subsection{Completed Course}

Let $S_{i}$ be a subcourse whose edges are assigned to the layers as follows: If variable $x_{i}$ does not occur in clause $c_{j}$ then both the $j^{\text {th }}$ reference and value edges are red and both the clamping edges of $S_{i}$ are blue. If variable $x_{i}$ occurs negated in $c_{j}$ then the $j^{\text {th }}$ reference edge is red, $j^{\text {th }}$ value edge is blue and both the clamping edges are blue. If variable $x_{i}$ occurs un-negated in $c_{j}$ then the $j^{t h}$ reference edge is blue, $j^{\text {th }}$ value edge is red and both the clamping edges are red.

A course $K_{i}$ is constructed recursively as follows:

1. $K_{1}$ is the subcourse $S_{1} . S_{1}$ is called the first subcourse of $K_{1}$.

2. $K_{i}$ is constructed from $K_{i-1}$ by identifying the $k^{t h}$ output vertex of the $i-1^{t h}$ subcourse $S_{i-1}$ of $K_{i-1}$ with the $k^{\text {th }}$ input vertex of subcourse $S_{i} . S_{i}$ is called the $i^{\text {th }}$ subcourse of $K_{i}$. For every $j$ such that $1 \leq j<i$, the $j^{t h}$ subcourse of $K_{i}$ is the $j^{\text {th }}$ subcourse of $K_{i-1}$

A completed course $G$ is constructed from a course $K_{n}$, a hurdle $H$, a starting location $L$ and an ending location $L^{\prime}$ as follows: Join the $j^{\text {th }}$ separation vertex of $L$ with the $j^{\text {th }}$ input vertex of the first subcourse of $K_{n}$ by a blue edge. Join the $j^{\text {th }}$ output vertex of the $n^{\text {th }}$ subcourse of $K_{n}$ with the $j^{\text {th }}$ input vertex of $H$ by a blue edge. Join the $j^{\text {th }}$ output vertex of $H$ with the $j^{\text {th }}$ separation vertex of $L^{\prime}$ by a blue edge. Fig. 11(a) shows a high level view of $G$.

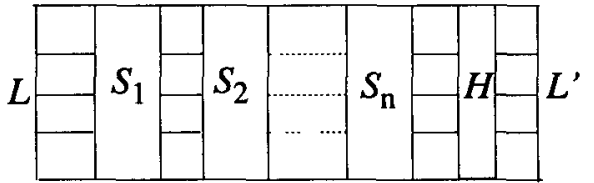

(a)

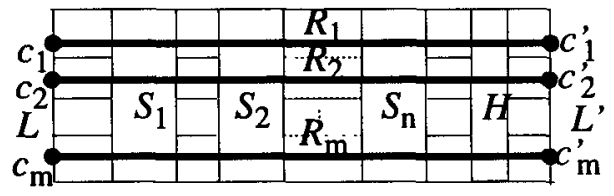

(b)

Figure 11: A high level view of $(a)$ Completed Course $G ;(b)$ angle graph $A$.

\subsection{Angle graph A}

Angle graph $A$ has a completed course $G$ as its (angle) subgraph. Let $K, H, L$ and $L^{\prime}$ be the course, hurdle, starting location and ending location respectively in $G$. In addition to $G$, for each clause $c_{j}$, graph $A$ has a runner $R_{j}$ with the $j^{\text {th }}$ starting and ending vertices of $L$ and $L^{\prime}$ respectively as its first and last end points. $R_{j}$ is called the representative of clause $c_{j}$ in $A$. This completes the construction of $A$. Fig. 11(b) shows a high level view of $A$.

Following correspondence exists between a biplanar drawing $\Delta$ of $A$ and a satisfiability assignment $\psi$ of $C$ : A variable $x_{i}$ is true in $\psi$ if and only if the subcourse $S_{i}$ is left heavy.

Theorem 7. $C$ has a satisfiability assignment if and only if the bilayered angle graph $A$ is biplanar.

Theorem 8. The problem of testing whether a bilayered angle graph is biplanar is NP-hard.

Corollary 9. The problem of testing whether a multilayered angle graph is multiplanar is NP-hard. 


\section{References}

1. R.F. Cohen, G. Di Battista, R. Tamassia, I.G. Tollis, and P. Bertolazzi, "A Framework for Dynamic Graph Drawing," Proc. ACM Symp. on Computational Geometry, 1992, pp. 261-270.

2. I.F. Cruz, A. Garg, "Drawing Graphs by Example Efficiently: Trees and Planar Acyclic Digraphs," Graph Drawing '94 (Proc. DIMACS Workshop on Graph Drawing), to appear.

3. G. Di Battista, P. Eades, R. Tamassia and I.G. Tollis, "Algorithms for Drawing Graphs: An Annotated Bibliography," Comp. Geom. Theory Appl., to appear

4. G. Di Battista, R. Tamassia, and I.G. Tollis, "Area Requirement and Symmetry Display of Planar Upward Drawings," Disc. \& Comp. Geom., vol. 7, 1992.

5. G. Di Battista and L. Vismara, "Angles of Planar Triangular Graphs," Proc. ACM Symp. on Theory of Computing, 1993.

6. P. Eades and T. Lin. "Algorithmic and declarative approaches to aesthetic layout," Graph Drawing '93 (Proc. ALCOM Workshop on Graph Drawing).

7. M. Formann, T. Hagerup, J. Haralambides, M. Kaufmann, F.T. Leighton, A. Simvonis, E. Welzl, and G. Woeginger, "Drawing Graphs in the Plane with High Resolution," Proc. FOCS, 1990, pp. 86-95.

8. A. Garg, M.T. Goodrich, and R. Tamassia, "Area-efficient Upward Tree Drawings", Proc. ACM Symp. on Computational Geometry, 1993, pp. 359-368.

9. A. Garg and R. Tamassia, "Planar Drawings and Angular Resolution: Algorithms and Bounds", European Symposium on Algorithms, 1994.

10. M.R. Gary and D.S. Johnson, "Computers and Intractability: A Guide to the Theory of NP-Completeness," W.H. Freeman, New York, NY 1979.

11. M. Himsolt, "A View to Graph Drawing Algorithms Through GraphEd", Graph Drawing '93 (Proc. ALCOM Workshop on Graph Drawing), Paris, 1993.

12. T. Kamada. On Visualization of Abstract Objects and Relations. PhD thesis, Department of Information Science, University of Tokyo, 1988.

13. G. Kant. Drawing planar graphs using the lmc-ordering. In Proc. 33th Annu. IEEE Sympos. Found. Comput. Scz., pages 101-110, 1992.

14. C.E. Leiserson, "Area-Efficient Graph Layouts (for VLSI)," Proc. IEEE Symp. on Foundations of Computer Science, 1980, pp. 270-281.

15. S.M. Malitz and A. Papakostas, "On the Angular Resolution of Planar Graphs," Proc. ACM Symp. on Theory of Computıng, 1992, pp. 527-538.

16. R. Tamassia. On embedding a graph in the grid with the minimum number of bends. SIAM J. Comput., 16(3):421-444, 1987.

17. R. Tamassia and I. G. Tollis. A unified approach to visibility representations of planar graphs. Discrete Comput. Geom., 1(4):321-341, 1986.

18. L. Valiant, "Universality Considerations in VLSI Circuits," IEEE Trans. on Computers, vol. C-30, 1981, pp. 135-140.

19. G. Vijayan, "Geometry of Planar Graphs with Angles", Proc. ACM Symp. on Computational Geometry, 1986, pp. 116-124.

20. G. Vijayan and A. Wigderson, "Rectiliear Graphs and their Embeddings," SIAM Journal of Computing, Vol. 14, No. 2, May 1985. 